PROCEEDINGS OF THE

AMERICAN MATHEMATICAL SOCIETY

Volume 126, Number 9, September 1998, Pages 2677-2686

S 0002-9939(98)04319-6

\title{
EXACT ITERATIVE RECONSTRUCTION ALGORITHM FOR MULTIVARIATE IRREGULARLY SAMPLED FUNCTIONS IN SPLINE-LIKE SPACES: THE $L^{p}$-THEORY
}

\author{
AKRAM ALDROUBI AND HANS FEICHTINGER \\ (Communicated by Palle E. T. Jorgensen) \\ Dedicated to the memory of Richard J. Duffin
}

\begin{abstract}
We prove that the exact reconstruction of a function $s$ from its samples $s\left(x_{i}\right)$ on any "sufficiently dense" sampling set $\left\{x_{i}\right\}_{i \in \Lambda}$ can be obtained, as long as $s$ is known to belong to a large class of spline-like spaces in $L^{p}\left(\mathcal{R}^{n}\right)$. Moreover, the reconstruction can be implemented using fast algorithms. Since a limiting case is the space of bandlimited functions, our result generalizes the classical Shannon-Whittaker sampling theorem on regular sampling and the Paley-Wiener theorem on non-uniform sampling.
\end{abstract}

\section{INTRODUCTION}

In sampling theory, the main goal is to reconstruct a continuous function $g \in$ $C\left(\mathcal{R}^{n}\right)$ from its samples $g\left(x_{i}\right)$ on a sampling set $X=\left\{x_{i}\right\}_{i \in \Lambda} \subset \mathcal{R}^{n}$, where $\Lambda$ is a countable indexing set, e.g., $\Lambda=\mathcal{Z}$. If the sampling is uniform, i.e., the set $X=\left\{x_{i}\right\}_{i \in \Lambda} \subset \mathcal{R}^{n}$ lies on a uniform Cartesian grid, then the function $g(x)$ can be recovered exactly from its samples as long as $g \in L^{2}(\mathcal{R})$ is bandlimited and the grid-points density is larger than the Nyquist density [20]. This is the classical Shannon-Whittaker sampling theorem. In particular, if $g \in L^{2}(\mathcal{R})$ and its Fourier transform $\hat{g}$ is such that $\hat{g}(f)=0, \forall f \notin I=\left[-\frac{1}{2}, \frac{1}{2}\right]$ (i.e., $g \in B_{\frac{1}{2}}$ ), then $g$ can be recovered from $g\left(x_{0}+k\right), k \in \mathcal{Z}$, by the interpolation formula [20], [23]

$$
g(x)=\sum_{k \in \mathcal{Z}} g\left(x_{0}+k\right) \operatorname{sinc}\left(x-x_{0}-k\right),
$$

where $x_{0} \in \mathcal{R}$ is arbitrary, and where, the interpolating function $\operatorname{sinc}(x)=\frac{\sin (\pi x)}{\pi x}$ is simply the inverse Fourier transform of the ideal filter function $\chi_{I}(f)$ in the band $I=\left[-\frac{1}{2}, \frac{1}{2}\right]$ (i.e., $\chi_{I}(f)$ is the characteristic function of the interval $I: \chi_{I}(f)=$ $1, \forall f \in I$, and $\left.\chi_{I}(f)=0, \forall f \notin I\right)$. A statement identical to the ShannonWhittaker sampling theorem is that the set of bandlimited functions $B_{\frac{1}{2}}$, with spectrum in $\left[-\frac{1}{2}, \frac{1}{2}\right]$, is precisely the set of functions belonging to the space $S^{2}$ (sinc)

Received by the editors January 28, 1997.

1991 Mathematics Subject Classification. Primary 42C15, 46A35, 46E15, 46N99, 47B37.

Key words and phrases. Non-uniform sampling, shift-invariant spaces, Riesz basis.

This research was partially supported through the FWF-project S-7001-MAT of the Austrian Science Foundation.

(C) 1998 American Mathematical Society 
given by

$$
S^{2}(\sin c)=\left\{\sum_{k \in \mathcal{Z}} c(k) \operatorname{sinc}(x-k) \mid c \in \ell^{2}\right\} .
$$

In fact, the set $\{\operatorname{sinc}(x-k)\}_{k \in \mathcal{Z}}$ is an orthonormal, hence an unconditional basis for the space $B_{\frac{1}{2}}$. Recall that a basis $\left\{e_{k}\right\}_{k \in \mathcal{Z}}$ of a Banach space $\mathcal{B}$ is unconditional if $\sum_{k} c(k) e_{k} \in \mathcal{B}$ implies that $\sum_{k} \epsilon_{k} c(k) e_{k} \in \mathcal{B}$ for any choice of $\epsilon_{k}$ equal to either +1 or -1 [17, pp. 16].

The Shannon-Whittaker sampling theorem can be generalized by changing the sinc-function in (1.2) to an appropriate generating function $\lambda(x)$ as in [2], [3], [4]:

$$
S^{2}(\lambda)=\left\{\sum_{k \in \mathcal{Z}} c(k) \lambda(x-k) \mid c \in \ell^{2}\right\} .
$$

In this generalization, $\lambda$ does not have to be band-limited, hence $S^{2}(\lambda)$ contains functions that are not bandlimited. The bandlimited case and the expansion (1.2) are obtained as the special case $\lambda(x)=\operatorname{sinc}(x)$. Moreover, the sampling theory for bandlimited functions is also a limit case for families $\lambda^{n}(x)$ with increasing smoothness as $n \rightarrow \infty$ (see [4] for details, and [5], [22] for examples that use polynomial spline functions). Other generalizations of the uniform sampling theory are discussed in [14], [15].

The classical result for non-uniform sampling is due to Paley and Wiener, and it states that if the set $X=\left\{x_{i}\right\}_{i \in \mathcal{Z}} \subset \mathcal{R}$ is such that $\left|x_{i}-i\right|<\pi^{-2}$, then a bandlimited function $g \in C(\mathcal{R})$, with spectrum in $[-\gamma, \gamma]$ for some $|\gamma|<\frac{1}{2}$, can be completely recovered from its samples $g\left(x_{i}\right)$ [19]. Duffin and Eachus showed that the same is true if $\left|x_{i}-i\right| \leq 0.22$ [7], and Kadec showed that the maximal upperbound constant is 0.25 [16]. A detailed exposition and other generalizations of the Paley-Wiener irregular sampling result can be found in [6]. There are also other types of results on irregular sampling in which the sampling set is non-uniform, but associated with the space of functions in a specific way [24], [25]. However, such results are different from those of Paley-Wiener, which do not require a fixed sampling set, but any sufficiently dense sampling set.

Extension to the multidimensional irregular sampling of bandlimited functions in $L^{p}$-spaces can be found in [10], [13]. The results are based on the properties of Wiener amalgam spaces [9], [10], which we also use in this paper. In particular, we will use the Wiener spaces $W^{p}=W\left(C, L^{p}\right)$, which are locally continuous and globally $L^{p}$. Specifically, a continuous function $g$ belongs to $W^{p}$ if its localization

$$
F_{h}[g](x)=\sup _{\xi \in \mathcal{R}^{n}}|h(\xi-x) g(\xi)|
$$

by the window function $h(\xi-x) \in \mathcal{D}\left(\mathcal{R}^{n}\right)$ is globally $L^{p}$ (here, $\mathcal{D}\left(\mathcal{R}^{n}\right)$ is the usual space of compactly supported, $C^{\infty}$ test functions):

$$
\|g\|_{W^{p}}^{p}=\int_{\mathcal{R}^{n}}\left|F_{h}[g](x)\right|^{p} d x<\infty .
$$

It turns out that an equivalent characterization of Wiener amalgam spaces can be obtained by a discrete form of (1.5) that gives the equivalent norm

$$
\|g\|_{W^{p}}^{p} \approx \sum_{l \in \mathcal{Z}^{n}}\left|F_{h}[g](l)\right|^{p},
$$


e.g., if the set $\{h(x-l)\}_{l \in \mathcal{Z}^{n}}$ forms a Bounded Uniform Partition of Unity (BUPU), i.e., $\sum_{l} h(x-l)=1[8]$. An important feature of both descriptions of these spaces is the fact that they do not depend on the choice of the window function $h(x) \in$ $\mathcal{D}\left(\mathcal{R}^{n}\right)$, i.e., changing $h$ will simply change the norm of the spaces to an equivalent norm [11].

In the present paper, we will generalize the theory of multidimensional irregular sampling in [10], [13] to the case of spline-like spaces $S(\lambda)$ of the form

$$
S(\lambda)=\left\{s=\sum_{k \in \mathcal{Z}^{n}} c(k) \lambda(x-k)\right\} .
$$

Our results can also be viewed as a generalizations of those in [18], where some ideas from [10], [13], and ideas similar to those in [4] are used to construct, under restrictive conditions, an irregular sampling theory for polynomial spline and other wavelet subspaces of $L_{2}(\mathcal{R})$.

Since the space $S(\lambda)$ that we consider must belong to $L^{p}\left(\mathcal{R}^{n}\right)$, the function $\lambda$ cannot be chosen arbitrarily. Moreover, we want the set $\{\lambda(x-k)\}_{k \in \mathcal{Z}^{n}}$ to form an unconditional basis of $S(\lambda)$. A sufficient condition is given by:

Proposition 1.1. The space $S(\lambda)$ is a closed subspace of $L^{p}(1 \leq p<\infty)$, and the set $\{\lambda(x-k)\}_{k \in \mathcal{Z}^{n}}$ forms an unconditional basis of $S(\lambda)$ if there exist two constants $B \geq A>0$ such that

$$
A\|c\|_{\ell^{p}} \leq\left\|\sum_{k \in \mathcal{Z}^{n}} c(k) \lambda(x-k)\right\|_{L^{p}} \leq B\|c\|_{\ell^{p}} .
$$

To see that $\{\lambda(x-k)\}_{k \in \mathcal{Z}^{n}}$ is a basis, simply note that if $\sum_{k \in \mathcal{Z}^{n}} c(k) \lambda(x-k)=0$, then the left inequality of (1.8) implies that $c=0$. The inequality on the right of (1.8) implies that this basis is unconditional. For the special case of $L^{2}\left(\mathcal{R}^{n}\right)$, to satisfy (1.8), a necessary and sufficient condition on a function $\lambda$ is that the Fourier transform $\hat{a}(f)=\sum_{k \in \mathcal{Z}^{n}}|\hat{\lambda}(f+k)|^{2}$ of the sampled autocorrelation $a(k)=$ $\left(\lambda * \lambda^{\vee}\right)(k), k \in \mathcal{Z}^{n}$, must be uniformly bounded above and below (here by definition $\left.\lambda^{\vee}(x)=\lambda(-x)\right)$, i.e., there exist two constants $M \geq m>0$ such that

$$
m \leq \hat{a}(f)=\sum_{k \in \mathcal{Z}^{n}}|\hat{\lambda}(f+k)|^{2} \leq M \quad \text { a.e. }
$$

This result can be found in [3], [4], and is also a special case of a general result in [1].

We are now ready to give a formal definition of the spline-like spaces $S^{p}(\lambda)$ that we will use for the remainder of this paper:

Definition 1.1. For $1 \leq p<\infty$, a spline-like space $S^{p}(\lambda)$ is a space described by (1.7) with $c \in \ell^{p}$, and where $\lambda$ satisfies condition (1.8), hence $S^{p} \subset L^{p}$.

\section{MAin Results}

Our ability to reconstruct a function $g \in S(\lambda)$ from irregularly spaced samples depends on the sampling density of the sampling set. The density measure introduced below (see [11], [12]) will play the same role for irregular sampling as the Nyquist rate for the uniform sampling case. 
Definition 2.1. A set $X=\left\{x_{i}\right\}_{i \in \Lambda}$ is $\gamma$-dense in $\mathcal{R}^{n}$, if $\mathcal{R}^{n}$ is the union of balls centered on $x_{i}$, and of radius $\gamma$ :

$$
\mathcal{R}^{n}=\bigcup_{i \in \Lambda} B_{\gamma}\left(x_{i}\right)
$$

If the set $X=\left\{x_{i}\right\}_{i \in \Lambda}$ is $\gamma$-dense, then we can always construct a piecewiseconstant function $V_{X} s$ that interpolates the samples $s\left(x_{i}\right)$ of a continuous function $s \in S^{p}$, i.e., $\left(V_{X} s\right)\left(x_{i}\right)=s\left(x_{i}\right), \forall i \in \Lambda$. The interpolating function that we construct is constant on the Voronoi domains $V_{i}$ of the sampling points $x_{i}$, defined as

$$
V_{i}:=\left\{x:\left|x_{i}-x\right|<\left|x_{j}-x\right| \forall i \neq j\right\} .
$$

Thus, for $s \in W^{p}$, we define the piecewise-constant interpolant operator $V_{X}$ by

$$
V_{X} s=\sum_{i \in \Lambda} s\left(x_{i}\right) \chi_{V_{i}}
$$

where $\chi_{V_{i}}$ is the characteristic function of $V_{i}$. It is well-known that the operator $V_{X}$ maps the space $W^{p}$ into the space $W\left(L^{\infty}, L^{p}\right) \subset L^{p}[8]$.

By interpolating the samples of a function $s \in S^{p}$ with $V_{X}$, and then projecting the interpolated function $V_{X} s$ on the space $S^{p}(\lambda)$, we get an approximation $s_{1} \in S^{p}$ of our original function $s$. Since $s$ and $s_{1}$ belong to $S^{p}$, the error $e=s-s_{1}$ belongs to $S^{p}$ as well. The values $e\left(x_{i}\right)=s\left(x_{i}\right)-s_{1}\left(x_{i}\right)$ of the error at the sampling points can be evaluated. Using the samples $e\left(x_{i}\right)$, we can repeat the interpolation and projection procedure to obtain a function $e_{1} \in S^{p}$. We add $e_{1}$ to $s_{1}$ to obtain the new approximation $s_{2}$ to our function $s$. By repeating this procedure, we obtain a sequence $s_{1}+e_{1}+e_{2}+\cdots$ that converges to $s$ as stated in the theorem below.

Theorem 2.1. Given $\lambda$ in $W^{1}$, and any bounded projector $P$ from $L^{p}$ onto $S^{p}(\lambda)$, there exists $\gamma>0(\gamma=\gamma(\lambda, p, P))$ such that any $s \in S^{p}(\lambda)$ can be recovered from its samples $\left\{s\left(x_{i}\right)\right\}$ on any $\gamma$-dense set $X=\left\{x_{i}\right\}_{i \in \Lambda}$ by the following iterative algorithm:

$$
\left\{\begin{aligned}
s_{n+1} & =P V_{X}\left(s-s_{n}\right)+s_{n} \\
s_{0} & =P V_{X} s
\end{aligned}\right.
$$

Convergence of $s_{n}$ to $s$ occurs in the $W^{p}$-norm, hence in the $L^{p}$-norm and in the sup-norm (uniform convergence), and we have $\left\|s-s_{n}\right\|_{L^{p}} \leq\left\|s-s_{n}\right\|_{W^{p}} \leq C_{1} \alpha^{n}$ for some $\alpha=\alpha(\gamma)<1$, and $C_{1}<\infty$.

The iterative algorithm can be described equivalently by

$$
s_{n+1}=\left(I+T+T^{2}+\cdots+T^{n}\right) P V_{X} s
$$

where $T=I-P V_{X}$, and gives the inversion of $I-T$ by the Neumann series:

$$
(I-T)^{-1}=I+T+T^{2}+\cdots
$$

Remark 2.1. The fact that the sampling values $s\left(x_{i}\right)$ are well defined follows from the fact that, under our condition on the generating function $\lambda$, all the functions in the space $S^{p}(\lambda)$ are continuous.

Remark 2.2. By choosing the projector $P$ appropriately, the algorithm will not depend on the choice or knowledge of the operating space $L^{p}$. All we need to know is that the function $s$ is a spline-like function to assure us that the convergence will automatically be in the $L^{p}$-norm, whenever the function is in $S^{p} \subset L^{p}$. 
Remark 2.3. An important point is that the reconstruction does not depend on any individual sampling set $X$, but only on the their $\gamma$-density. This means that as long as the holes between samples is not too large, we can always recover any function in $S^{p}$ exactly with our iterative algorithm. Moreover, in contrast to other approaches, our method can handle clusters in the sampling set. On the other hand it is not a simple frame algorithm, since we are not in a Hilbert space setting anymore, and thus the alternative tools, such as Wiener amalgam spaces, have to be used.

Remark 2.4. The interpolant $V_{X}$ can be replaced by other types of (quasi)-interpolant without changing the validity of the theorem. In particular, we can choose a general BUPU $\Phi=\left\{\phi_{i}\right\}_{i \in \Lambda}$ satisfying: (1) $0 \leq \phi_{i} \leq 1, \forall i \in \Lambda$, (2) support $\phi_{i} \subset B_{\gamma}\left(x_{i}\right)$, (3) $\sum_{i \in \Lambda} \phi_{i}=1$, and use a (quasi)-interpolation of the form

$$
Q_{\Phi} s=\sum_{i \in \Lambda} s\left(x_{i}\right) \phi_{i}
$$

Theorem 2.1 relies on the theorem below. The proofs of both theorems are postponed to Section 3 .

Theorem 2.2. For $\lambda \in W^{1}$ one has:

(1) The space $S^{p}$ is continuously embedded in $W^{p}$, and the $L^{p}$-norm and the $W^{p}$ norm are equivalent for $S^{p}$, i.e., there exist two positive constants $C_{1}>0$ and $C_{2}>0$ such that

$$
C_{1}\|s\|_{W^{p}} \leq\|s\|_{L^{p}} \leq C_{2}\|s\|_{W^{p}} \quad \forall s \in S^{p} .
$$

(2) $\forall \epsilon>0$ there exists $\delta_{0}>0$ such that

$$
\left\|\operatorname{osc}_{\delta}(s)\right\|_{L^{p}} \leq \varepsilon\|s\|_{L^{p}} \quad \forall s \in S^{p}, \forall \delta \leq \delta_{0},
$$

where the oscillation function $\operatorname{osc}_{\delta}(s)$ is defined by

$$
\left(\operatorname{osc}_{\delta} s\right)(x)=\sup _{|y| \leq \delta}|s(x)-s(x+y)| .
$$

If the sampling set $X=\left\{x_{i}\right\}_{i \in \Lambda}$ in Theorem 2.1 is relatively separated (see [10] for the definition), and so $X$ has no cluster points (a set is relatively separated if it is separated, i.e., $x_{i}-x_{j} \geq h_{\min }>0, i \neq j$ ), then as a corollary of (2.6) in Theorem 2.2 and [10, Theorem 14 ] we obtain

Corollary 2.3. If the set $X=\left\{x_{i}\right\}_{i \in \Lambda}$ in Theorem 2.1 is relatively separated, then, for any $1 \leq p<\infty$, there exists a constant $C$ such that

$$
\left(\sum_{i \in \Lambda}\left|s\left(x_{i}\right)\right|^{p}\right)^{1 / p} \leq C\|s\|_{W^{p}} .
$$

Moreover, a function $s$ in $S(\lambda)$ belongs to $S^{p}(\lambda)$ if and only if the sequence $\left\{s\left(x_{i}\right)\right\}$ belongs to $\ell^{p}$. Hence the norm $\|s\|_{W^{p}}$ is equivalent to the norm $\left\|\left\{s\left(x_{i}\right)\right\}\right\|_{l^{p}}$.

Remark 2.5. In view of Corollary 2.3, if the sampling set $X$ is relatively separated, then one can decide if $s \in S$ belongs to $L^{p}$ from its sample values at $X$ alone. 
2.1. A special case. Within the $L^{2}$-theory, we can choose $P$ to be the orthogonal projection on $S^{2}$. For this case, the orthogonal projection can be obtained by a pre-filtering (or equivalently a convolution operation), followed by a sampling and finally a postfiltering. Specifically, the orthogonal projection $P$ of a function $g \in L^{2}\left(\mathcal{R}^{n}\right)$ on $S^{2}$ is given by [4]

$$
\begin{aligned}
P g & =\sum_{k \in \mathcal{Z}^{n}}\langle g, \stackrel{\circ}{\lambda}(x-k)\rangle \lambda(x-k) \\
& =\downarrow_{1}[g * \stackrel{\circ}{\lambda}] * \lambda
\end{aligned}
$$

where $\downarrow_{1}[\bullet]$ is the sampling operator with sampling step 1 , and where $\grave{\lambda} \in S^{2}$ is the dual function given in terms of the convolution inverse of $a(k)=\left(\lambda * \lambda^{\vee}\right)(k)$ by the linear combination formula [4]

$$
\grave{\lambda}=\sum_{k \in \mathcal{Z}^{n}} a^{-1}(k) \lambda(x-k) .
$$

All the operations involved in computing the orthogonal projection $\mathrm{Pg}$ in (2.10) consist of simple convolutions and additions only. This feature is often useful for signal processing and numerical analysis because it allows for fast "filtering" implementations. This filtering algorithm together with the fixed-point iterative algorithm (2.4) give rise to a fast reconstruction procedure.

Instead of the orthogonal projection $P$, we can also choose the oblique projection $P_{S_{1}^{2} \perp S_{2}^{2}}$ on $S_{1}^{2}\left(\lambda_{1}\right)$ in a direction orthogonal to a space $S_{2}^{2}\left(\lambda_{2}\right)$ [1], [21]. The only change between this case and the orthogonal projection is that the dual function $\AA$ in $(2.11)$ is now replaced by the function $\dot{\lambda}_{12}$ given by

$$
\stackrel{\circ}{\lambda}_{12}(x)=\sum_{k \in \mathcal{Z}^{n}} a_{12}^{-1}(k) \lambda_{1}(x-k),
$$

where $a_{12}(k)=\left(\lambda_{1} * \lambda_{2}^{\vee}\right)(k)$ is the sampled cross-correlation between the generating functions $\lambda_{1}$ and $\lambda_{2}$ of $S_{1}^{2}$ and $S_{2}^{2}$, respectively [21].

\section{Proof of the Theorems}

\subsection{Proof of Theorem 2.2.}

Proof. Let $\mathcal{D}\left(\mathcal{R}^{n}\right)$ denote the usual Schwartz space of compactly supported, $C^{\infty}\left(\mathcal{R}^{n}\right)$ test functions. We choose a bounded uniform partition of unity $\left\{h_{r}(x-l\}_{l \in \mathcal{Z}^{n}}\right.$ generated by a function $h_{r}(x) \in \mathcal{D}\left(\mathcal{R}^{n}\right)$ such that $\left|h_{r}(0)\right|=1,\left|h_{r}(x)\right| \leq|h(0)|, \forall x \in$ $\mathcal{R}^{n}$, and $h_{r}(x)=0$ for $|x| \geq r$. For $s \in W^{p}$ we have the pointwise estimate

$$
|s(\xi)|=\left|h_{r}(0) s(\xi)\right| \leq \sup _{x \in \mathcal{R}^{n}}\left|h_{r}(x-\xi) s(\xi)\right|=F_{h_{r}}[s](\xi) .
$$

The estimate (3.1) combined with the definitions (1.4) and (1.5) yields $\|s\|_{L^{p}} \leq$ $\left\|F_{h_{r}}[s]\right\|_{L^{p}}=\|s\|_{W^{p}}$, i.e., a continuous embedding of $W^{p}$ in $L^{p}$. This is a well-known fact (see [8]), but we have chosen to include the proof here for completeness. Thus, to finish the proof of part (1), all we need to show is that $S^{p} \subset W^{p}$, and that the inclusion is a continuous embedding. If $\lambda \in W^{1}$, then $\lambda \in W^{p}$ [8]. Let $h_{r} \in \mathcal{D}\left(\mathcal{R}^{n}\right)$ 
be chosen as in the beginning of this proof. For a function $s(x)=\sum c(k) \lambda(x-k)$, and any $l \in \mathcal{Z}^{n}$, we have

$$
\begin{aligned}
F_{h_{r}}[s](l) & =\sup _{x \in \mathcal{R}^{n}}\left|h_{r}(x-l) s(x)\right| \\
& \leq \sum_{k \in \mathcal{Z}^{n}}|c(k)| \sup _{x \in \mathcal{R}^{n}}\left|h_{r}(x-l) \lambda(x-k)\right| \\
& \leq \sum_{k \in \mathcal{Z}^{n}}|c(k)| F_{h_{r}}[\lambda](l-k) .
\end{aligned}
$$

Since $\lambda$ belongs to $W^{1}$, we use the norm equivalence (1.6) of the Wiener spaces to conclude that the sequence $F_{h_{r}}[\lambda](k)$ belongs to the sequence space $l^{1}$, and that $\|\lambda\|_{W^{1}} \approx\left\|F_{h_{r}}[\lambda]\right\|_{l^{1}}$. From this fact, (1.6), and the last inequality of (3.2), we obtain

$$
\|s\|_{W^{p}} \leq C\|c\|_{\ell^{p}}\|\lambda\|_{W^{1}} .
$$

This last inequality together with the left inequality of (1.8) implies that $S^{p}$ is continuously embedded in $W^{p}$, which completes the proof of part (1).

To prove part (2), we consider the oscillation function $\operatorname{osc}_{\delta}(s)$ given by (2.8). For $s(x)=\sum c(k) \lambda(x-k)$, we have

$$
\begin{aligned}
\operatorname{osc}_{\delta}(s) & =\sup _{|y|<\delta}\left|\sum_{k \in \mathcal{Z}^{n}} c(k)(\lambda(x-k)-\lambda(x+y-k))\right| \\
& \leq \sum_{k \in \mathcal{Z}^{n}}|c(k)| \sup _{|y|<\delta}|\lambda(x-k)-\lambda(x+y-k)| \\
& \leq \sum_{k \in \mathcal{Z}^{n}}|c(k)| \operatorname{osc}_{\delta}(\lambda) .
\end{aligned}
$$

If $\operatorname{osc}_{\delta}(\lambda)$ belongs to $W^{1}$, then from (3.4), and using an argument similar to the one that led to $(3.3)$, we would get

$$
\left\|\operatorname{osc}_{\delta}(s)\right\|_{L^{p}} \leq \text { Const }\|c\|_{\ell^{p}}\left\|\operatorname{osc}_{\delta}(\lambda)\right\|_{W^{1}} .
$$

If, furthermore, $\left\|\operatorname{osc}_{\delta}(\lambda)\right\|_{W^{1}} \rightarrow 0$ as $\delta \rightarrow 0$, then we would deduce part (2) of the theorem. Thus, all we need to show is

(a) $\operatorname{osc}_{\delta}(\lambda)$ belongs to $W^{1}$;

(b) $\lim _{\delta \rightarrow 0}\left\|\operatorname{osc}_{\delta}(\lambda)\right\|_{W^{1}}=0$.

To show part (a), we simply note that

$$
\sup _{x \in \mathcal{R}^{n}}\left(\left|h_{r}(x-l)\right| \sup _{|y| \leq \delta}|\lambda(x+y)|\right) \leq \sup _{x \in R^{n}}\left|h_{r_{2}}(x-l) \lambda(x)\right|,
$$


where $h_{r_{2}} \in \mathcal{D}\left(\mathcal{R}^{n}\right)$ is chosen so that $h_{r_{2}}(x)=1$ for all $|x| \leq r+\delta$, and $h_{r}$ is chosen as in the beginning of this proof. This last inequality implies that

$$
\begin{aligned}
& \sup _{x \in \mathcal{R}^{n}}\left(\left|h_{r}(x-l)\right| \operatorname{osc} \delta(\lambda)(x)\right)=\sup _{x \in \mathcal{R}^{n}}\left(\left|h_{r}(x-l)\right| \sup _{|y| \leq \delta}|\lambda(x+y)-\lambda(x)|\right) \\
& \quad \leq \sup _{x \in \mathcal{R}^{n}}\left(\left|h_{r}(x-l)\right| \sup _{|y| \leq \delta}|\lambda(x+y)|\right)+\sup _{x \in \mathcal{R}^{n}}\left|h_{r}(x-l) \lambda(x)\right| \\
& \quad \leq 2 \sup _{x \in R^{n}}\left|h_{r_{2}}(x-l) \lambda(x)\right| .
\end{aligned}
$$

Since $\lambda$ belongs to $W^{1}$, it follows from (3.7) that $\operatorname{osc}_{\delta}(\lambda)$ belongs to $W^{1}$ as well.

To prove part (b), we note that, since $\operatorname{osc}_{\delta} \lambda$ belongs to $W^{1}$, the equivalent norm (1.6) implies that for any $\epsilon>0$, there exists an integer $L_{0}>0$ such that

$$
\sum_{l \geq|L|} \sup _{x \in \mathcal{R}^{n}}\left(\left|h_{r}(x-l)\right| \sup _{|y| \leq \delta}|\lambda(x+y)-\lambda(x)|\right)<\frac{\epsilon}{2} \quad \forall L \geq L_{0}, \forall \delta \leq \delta_{0} .
$$

Moreover, since $\lambda$ is continuous, we can choose $\delta_{0}$ so small that if $\delta \leq \delta_{0}$, then

$$
\begin{aligned}
\sup _{x \in \mathcal{R}^{n}} & \left(\left|h_{r}(x-l)\right| \sup _{|y| \leq \delta}|\lambda(x+y)-\lambda(x)|\right) \\
& =\sup _{x \in B_{r}(l)}\left(\left|h_{r}(x-l)\right| \sup _{|y| \leq \delta}|\lambda(x+y)-\lambda(x)|\right) \\
& \leq \frac{\epsilon}{2 L_{0}} .
\end{aligned}
$$

Combining (3.8) and (3.9), we immediately obtain that for any $\epsilon>0$, there exists a $\delta_{0}>0$ such that

$$
\left\|\operatorname{osc}_{\delta}(\lambda)\right\|_{L^{p}} \leq \epsilon \quad \forall \delta \leq \delta_{0}
$$

which completes the proof.

3.2. Proof of Theorem 2.1. We start with the following lemma.

Lemma 3.1. If $P$ is a projector, and if $X=\left\{x_{i}\right\}_{i \in \Lambda}$ is $\gamma$-dense, then the operator $I-P V_{X}$ from $S^{p}$ into $S^{p}$ is a contraction for sufficiently small $\gamma$.

Proof of Lemma 3.1. For $s \in S^{p}$, part (1) of Theorem 2.2 implies that $s$ also belongs to $W^{p}$. Therefore, the function $s$ is continuous and the sampling $s\left(x_{i}\right)$ is well defined for any point $x_{i}$. Since the interpolant operator $V_{X}$ is a bounded linear operator that maps $W^{p}$ into $W\left(L^{\infty}, L^{p}\right) \subset L^{p}$ [8], we also conclude that $V_{X} s$ belongs to $L^{p}$. Moreover, since the sampling set $X$ is $\gamma$-dense, the definition of $\operatorname{osc}_{\gamma}$ in (2.8) implies the pointwise estimate

$$
\left|\left(s-V_{X} s\right)(x)\right| \leq\left(\operatorname{osc}_{\gamma} s\right)(x) \quad \forall x \in \mathcal{R}^{n},
$$

and hence we conclude that

$$
\left\|s-V_{X} s\right\|_{L^{p}} \leq\left\|\left(\operatorname{osc}_{\gamma} s\right)\right\|_{L^{p}} .
$$


Using Theorem 2.2, we obtain

$$
\begin{aligned}
\left\|s-P V_{X} s\right\|_{L^{p}} & =\left\|P s-P V_{X} s\right\|_{L^{p}} \\
& \leq C_{P}\left\|s-V_{X} s\right\|_{L^{p}} \\
& \leq C_{P}\left\|\left(\operatorname{osc}_{\gamma} s\right)\right\|_{L^{p}} \quad(\text { by }(3.11)) \\
& \leq \epsilon C_{P}\|s\|_{L^{p}} \quad(\text { by }(2.7)) .
\end{aligned}
$$

By choosing $\gamma$ sufficiently small, we can force $\epsilon$ to be as small as we wish (by part (2) of Theorem 2.2). In particular, we choose $\gamma$ so that $\epsilon C_{P}<1$ to obtain a contraction.

Using this lemma, we are now ready to prove Theorem 2.1:

Proof of Theorem 2.1. From (2.4), the sequence $e_{n}=s-s_{n}$ must satisfy

$$
e_{n+1}=e_{n}-P V_{X} e_{n} .
$$

Thus, from Lemma 3.1, we conclude that, for $\gamma$ sufficiently small, we have

$$
\left\|e_{n+1}\right\|_{L^{p}} \leq \alpha\left\|e_{n}\right\|_{L^{p}}
$$

where $\alpha<1$. Therefore $\left\|e_{n}\right\|_{L^{p}} \rightarrow 0$, and the proof is complete.

\section{Concluding Remarks}

We have shown how to reconstruct spline-like functions exactly, from their irregular samples. These functions are not bandlimited, in general. The bandlimited theory is a limit case. The theory is valid for any dimension, and it generalizes the Paley-Wiener theory on non-uniform sampling. Theorem 2.1 shows that the reconstruction algorithm converges exponentially fast $\left(O\left(\alpha^{n}\right), \alpha<1\right)$ as the number of iterations $n$ increases. Since the contraction factor $\alpha$ is a decreasing function of the density $\gamma$, the algorithm will converge more rapidly for denser sets. For the case $L^{2}\left(\mathcal{R}^{n}\right)$, the orthogonal projection as well as some of the oblique projection operators can be implemented with a fast filtering algorithms as described in subsection 2.1. Therefore, for this case, we have a very efficient algorithm for the reconstruction which consists of the repetitive application of a (quasi)-interpolation followed by a filtering (equivalently a convolution).

\section{REFERENCES}

[1] A. Aldroubi. Oblique projections in atomic spaces. Proc. Amer. Math. Soc., 124:2051-2060, 1996. MR 96i: 42020

[2] A. Aldroubi and M. Unser. Families of wavelet transforms in connection with Shannon's sampling theory and the Gabor transform. In Wavelets: A Tutorial in Theory and Applications, C.K. Chui, ed., pages 509-528, Academic Press, San Diego, CA, 1992. MR 93a:94003

[3] A. Aldroubi and M. Unser. Families of multiresolution and wavelet spaces with optimal properties. Numer. Funct. Anal. and Optimiz., 14(5):417-446, 1993. MR 94j:42045

[4] A. Aldroubi and M. Unser. Sampling procedure in function spaces and asymptotic equivalence with Shannon's sampling theory. Numer. Funct. Anal. and Optimiz., 15(1):1-21, 1994. MR 95a:94002

[5] A. Aldroubi, M. Unser, and M. Eden. Cardinal spline filters: Stability and convergence to the ideal sinc interpolator. Signal Processing, 28:127-138, 1992.

[6] J.J. Benedetto. Irregular sampling and frames. In Wavelets: A Tutorial in Theory and Applications, C.K. Chui, ed., pages 445-507, Academic Press, San Diego, CA, 1992. MR 93c:42030

[7] R.J. Duffin and J.J. Eachus. Some notes on an expansion theorem of Paley and Wiener. Bull. Amer. Math. Soc., 48:850-855, 1942. MR 4:97e 
[8] H.G. Feichtinger. Banach convolution algebras of Wiener type. In Proc. Conf. Functions, Series, Operators, Budapest, pages 509-524, Amsterdam-Oxford-New York, 1980. Colloquia Math. Soc. J. Bolyai, North Holland Publ. Co. MR 85j:43005

[9] H.G. Feichtinger. Generalized amalgams, with applications to Fourier transform. Can. J. of Math., 42(3):395-409, 1990. MR 92f:46038

[10] H.G. Feichtinger. New results on regular and irregular sampling based on Wiener amalgams. In K. Jarosz, editor, Proc. Conf. Function Spaces, volume 136 of Lect. Notes in Pure and Appl. Math, pages 107-122, Edwardsville, IL, April 1990, 1991. Marcel Dekker. MR 93c: $46038 \mathrm{a}$

[11] H.G. Feichtinger. Wiener amalgams over Euclidean spaces and some of their applications. In K. Jarosz, editor, Proc. Conf. Function Spaces, volume 136 of Lect. Notes in Pure and Appl. Math., pages 123-137, Edwardsville, IL, April 1990, 1991. Marcel Dekker. MR 93c:46038b

[12] H.G. Feichtinger and K. Gröchenig. Non-orthogonal wavelet and Gabor expansions, and group representations. In Wavelets and their Applications, M.B. Ruskai, G. Beylkin, R.R. Coifman, I. Daubechies, S. Mallat, Y. Meyer, and L. Raphael, eds., pages 353-375, Jones and Bartlett, Boston, 1992. MR 93k:42017

[13] H.G. Feichtinger and K. Gröchenig. Iterative reconstruction of multivariate band-limited functions from irregular sampling values. SIAM J. Math. Anal. 231:244-261, 1992. MR 93a:94006

[14] J.R. Higgins. Five short stories about the cardinal series. Bull. Amer. Math. Soc., 121:45-89, 1985. MR 86k: 42045

[15] A.J. Jerri. The Shannon sampling theorem-its various extensions and applications: A tutorial review. Proc. IEEE, 65:1565-1596, 1977.

[16] M.I. Kadec. The exact value of the Paley-Wiener constant. Soviet Math. Doklady, 5:559-561, 1964. MR 28:5289

[17] J. Lindenstrauss and L. Tzafiri. Classical Banach Spaces. Springer-Verlag, Berlin, 1973. MR 54:3344

[18] Y. Liu. Irregular sampling for spline wavelet subspaces. IEEE Trans. Inform. Theory, 42(2):623-627, 1996. MR 97b:94006

[19] R.E.A.C Paley and N. Wiener. Fourier transform in the complex domain. Amer. Math. Soc. Colloquium publications. Amer. Math. Soc., 1934.

[20] C.E. Shannon. Communications in the presence of noise. Proc. IRE, 37:10-21, 1949. MR 10:464e

[21] M. Unser and A. Aldroubi. A general sampling theory for non-ideal acquisition devices. IEEE Trans. on Signal Processing, 42(11):2915-2925, 1994.

[22] M. Unser, A. Aldroubi, and M. Eden. Polynomial spline signal approximations: filter design and asymptotic equivalence with Shannon's sampling theorem. IEEE Trans. Image Process., 38:95-103, 1991. CMP 92:07

[23] J.M. Whittaker. Interpolation Function Theory. Cambridge University Press, London, 1935.

[24] K. Yao. Application of Reproducing Kernel Hilbert Spaces-bandlimited signal models. Information and Control, 11:429-444, 1967.

[25] A.I. Zayed. On Kramer's sampling theorem associated with general Sturm-Liouville problems and Lagrange interpolation. SIAM J. Appl. Math., 51:575-604, 1991. MR 92b:34038

National Institutes of Health, Biomedical Engineering and Instrumentation ProGram, Bethesda, Maryland 20892

E-mail address: aldroubi@helix.nih.gov 37240

Current address: Department of Mathematics, Vanderbilt University, Nashville, Tennessee

E-mail address: aldroubi@math.vanderbilt.edu

University of Vienna, Department of Mathematics, Strudlhofg. 4, A-1090 Wien, Austria

E-mail address: fei@tyche.mat.univie.ac.at 